\title{
Introduction: the Josephson Effect and Its Role in Physics
}

\author{
Francesco Tafuri $^{1}$
}

Accepted: 3 December 2020 / Published online: 27 January 2021

(C) The Author(s), under exclusive licence to Springer Science+Business Media, LLC part of Springer Nature 2021

It has been a real honor for me to organize this special issue which celebrates Prof. Brian Josephson's 80th birthday and his wonderful discovery [1, 2]. I am grateful to Sara Kate Heikerott, Senior Editor, and to Vladimir Kresin, Editor of the Journal of Superconductivity and Novel Magnetism, for the invitation. I am also grateful to all the authors for their contributions, and, especially, to John Clarke, who was playing an important role in the beginning of the project with his help with the content of the issue. It has been a real privilege for me to be in contact with such great and inspiring colleagues and friends. This issue obviously builds on the already available impressive literature including some dedicated volumes [3-8] and tries to give a flavor of the amazing impact of the Josephson effect in so many different fields of fundamental research and applications.

I have been asked to give a short overview in view of a possible unitary vision over the various contributions. I would start from the contributions of John Clarke and John Rowell respectively. Apart from the scientific message, they are amazing historical documents. John Clarke in his "Brian Josephson and the Royal Society Mond Laboratory" brings us back to the autumn of 1964 in the Royal Society Mond Laboratory in Cambridge. We are at the Mond seminar of Brian Josephson, who is explaining his theory of pair tunneling in 1962 and its experimental demonstration by Anderson and Rowell during the following year, and finally observation of quantum interference by Jaklevic et al. - the introduction of the dc SQUID (Superconducting QUantum Interference Device). John's conclusions are a wonderful message not only for Brian Josephson but for every student looking for a project: "Looking back, it all started 56 years ago when I heard Brian Josephson's seminar and Brian Pippard's suggestion of the voltmeter the very next day. Brian, I offer my very best wishes and congratulations on

Francesco Tafuri

francesco.tafuri@unina.it

1 Dipartimento di Fisica E.Pancini, Universitá di Napoli Federico II, Complesso Universitario di Monte Sant'Angelo, Via Cinthia, 21, 80126, Naples, Italy your 80th birthday. Thank you for your extraordinary insight that created my career."

John Rowell's contribution is a wonderful piece of physics' history. He goes back to the summer of 1962 , where rumors of Josephson's proposal of "possible new effects" began to circulate at Bell Labs and became more specific with the return of Phil Anderson from his year at Cambridge. John recalls that "They were not met with universal acceptance, especially by theorists!" Inspired by the fabrication processes described by Ivar Giaever, he began to make $\mathrm{Al} / \mathrm{AlOx} / \mathrm{Pb}$ junctions in 1962 and on January 3 of 1963, he decided to measure one such junction in the only cryostat at Bell that reached well below $1 \mathrm{~K}$. The rest of the story is in his contribution including sketches on the conversations with Phil Anderson, and two exciting original pictures from his lab notebook with the very first observation of the Josephson effect.

Kristian Fossheim gives a few historical remarks on Brian Josephson and his discovery. He gently retraces some key historical steps on the meaning and the impact of his discovery, which are more extensively discussed in his book on "Discoveries and Discoverers in Superconductivity" published by Springer.

Alex Braginski revisits beneficial consequences of the Josephson effect for science and economy, with a special focus on the impact of Josephson devices and circuits in many areas of science and in geophysical prospecting for minerals. Alex mostly focuses on the SQUIDs, and expects a very large social potential in terms of benefits to patients thanks to a broad adoption of contactless noninvasive medical diagnostics using SQUIDs.

Kent Irwin and Hsiao-Mei Sherry Cho discuss the combined use of SQUIDs and transition edge sensors (TES) for cosmic microwave background (CMB) experiments. SQUIDs readout for constant voltage biased TES enables the detection of the infinitesimal fluctuation in CMB, carrying abundant information on how the universe began, what it is made of, and how it evolved to its current state. These are great additional examples of how powerful Josephson junctions can be as direct or indirect sensors in a variety of experiments in very different fields. 
Cathy Foley and her group at CSIRO give another remarkable example of the use of the Josephson effect in SQUIDS. Here high critical temperature superconductors (HTS) are used taking advantage of the special property of HTS grain boundaries forming Josephson junctions (JJs). SQUIDs are employed in a system used for mineral exploration and have been successful in supporting the delineation and discovery of many billions of dollars of ore bodies. Cathy and her team also describe how Josephson junctions can both detect and create high frequency radiation using the incredible bandwidth as a result of the large band gap associated with HTS materials, lending themselves to a wide range of high frequency receivers and detector systems.

HTS JJs have a special place also in the contribution of Hans Hilgenkamp, who deals with Josephson information processing and storage technologies, and specifically with Josephson memories. Hans sketches most achievements in the field from very large scale Integrated Josephson junction-based Random Access Memories to the Resistive/ Rapid Single Flux Quantum (RSFQ)-technology. Departing from the "latching logic" that employs switching between the superconducting and voltage states of hysteretic Josephson junctions, the RSFQ circuits use flux quantization in superconducting loops. Single magnetic flux-quanta can enter or leave superconducting loops by means of the $2 \pi$ phase slip associated with a single Josephson oscillation, induced by a current/voltage pulse. $\pi$-shift Josephson memory devices can be made of HTS JJs taking advantage of the d-wave order parameter symmetry or of a $\mathrm{JJ}$ based on a ferromagnetic barrier. The last example of the use of JJs for information processing comes from qubit - implementations, in the quest to realize quantum computers.

On the same line, Theodore Van Duzer resumes the international effort to build a digital computer that would surpass what could be done with semiconductors. He with his team in Berkeley was aiming at making a hybrid Josephson junction-CMOS miniature computer that would take advantage of what each technology could do best, superconductors for logic and semiconductors for memory. They were finally able to demonstrate the operation of an hybrid system using commercial CMOS for the memory.

Ferromagnetic Josephson junctions are of inspiration for Mark Blamire for more general considerations on the potential of the Josephson energy. More specifically in the context of magnetic Josephson junctions the Josephson energy offers a potential mechanism for controlling magnetic memory devices. Marks also has memories of the lectures from Brian Josephson in Cambridge.

Michel Devoret's contribution is a wonderful insight on the notion of "Brian Josephson's gauge-invariant phase difference." His conclusion is that the topology of a variable in quantum mechanics is relative to the constraints/ interactions to which this variable is submitted by the various parts of the system. Whether the Josephson's gaugeinvariant phase difference lives "on a line, or a circle" is not universal, it depends on the type of circuit in which the junction is embedded. This aspect is of great relevance for "Circuit Quantum Electrodynamics," which is currently a very hot topic in the realization of quantum computers. The Josephson junctions play as usual key role in the realization of superconducting qubits and quantum circuits. Major surprises are expected in multi-degree-of-freedom circuits that defy our classical imagination. Michel stresses on the spectacular and paradoxical precision of the Josephson effect, despite the fact that a Josephson junction can be very disordered at the microscopic level. That's the feeling that each of us has when looking at the structural investigations of the fabricated junction. There is always a magic balance between the inner macroscopic coherence of a Josephson junction and the "dirt" at the microscopic level. And this may also happen junctions using HTS (see below)!

Also in Michel's piece, there is a reference to an inspiring moment during his graduate studies, specifically a lecture that Brian Josephson was giving in 1976 at the 26th Lindau Nobel Laureate Meeting, in company of Paul Dirac, Felix Bloch, Leon Cooper and Ivar Giaver. Six years later, when he joined Professor John Clarke's laboratory for a postdoc at Berkeley in 1982, Michel came to appreciate how extraordinary was the gift Brian Josephson had given to the entire physics community.

Saro Fazio in his note highlights the importance of the Josephson effect in the realization of quantum simulators, thus its pivotal role also in the emerging field of quantum technologies. Josephson Junction Arrays (JJAs), a regular network of superconducting islands weakly coupled by means of tunnel junctions, were originally used to study the properties of classical models of statistical mechanics, and towards the end of 1980s Josephson arrays allowed to investigate quantum phase transitions in a controlled fashion. Saro discusses how superconducting networks, both Josephson junction arrays and more recently cavity arrays, turned out to be excellent experimental platforms to simulate static and dynamical phenomena of quantum manybody systems.

The role of the Josephson effect in studying order parameter symmetry has been of great relevance for HTS. This has been touched by the manuscript of Hans Hilgenkamp and will be also partly described here. Another impressive example comes from $\mathrm{Sr}_{2} \mathrm{RuO}_{4}$ (SRO), as discussed by Anthony J. Leggett and Ying Liu. It was conjectured that SRO order parameter (OP) has a form similar to that realized in the superfluid phases of 3-He, namely, odd parity and spin triplet. Experiments based on Josephson junctions 
show that the pairing symmetry in SRO cannot be of the even parity, spin singlet type, and basically excluding all bulk p-wave states, and are apparently in contradiction with other experiments. Tony and Ying present a comprehensive approach, aimed at resolving this primafacie contradiction, taking into account implications of other important experiments on SRO, including that of the muon spin rotation.

Another example of the Josephson effect as a key for other central issues in condensed matter physics is given by the manuscript of Yukio Tanaka and Shun Tamura, who deal with the theoretical achievements on the physics of surface Andreev bound state and odd-frequency pairing. The study of zero energy surface Andreev bound state, started in the context of the pairing symmetry of HTS cuprates, is of great relevance for topological superconductors with Majorana edge state, which is currently a key theme in condensed matter physics.

The other contributions deal with very special systems where Josephson coupling can take place.

Reinhold Kleiner describes coupling between planes in HTS crystals; Reinhold's contribution reports on how himself and Paul Muller inspired by a theoretical paper entitled BCS versus Josephson pair hopping between the $\mathrm{CuO}_{2}$ layers in HTS were able to demonstrate that BSCCO single crystals act as stacks of "intrinsic" Josephson junctions (IJJs) by performing out-of-plane transport experiments. The inspiration behind the experiment was the feeling that the Josephson effect is really everywhere in the cuprates. Also in Reinhold's career, as in most of the contributors, the Josephson effect is everywhere.

Guy Deutscher describes granular superconductivity with Josephson coupling between grains. He also discusses how the Kondo effect can overcome the Coulomb blockade.

Vladimir Kresin and Yurii Ovchinnikov describe the impact of size quantization and nano-based Josephson tunneling networks. For Vladimir and Yuri Josephson networks are the ideal tool to create the macroscopic system capable of transferring the superconducting current, as a result of the combined effect of the spectrum's shell structure and of the Josephson tunneling. According to their predictions, this system is capable of transferring the superconducting current at high temperatures, potentially, at room temperature. Synchronization of the frequencies of the various junctions would occur and been confirmed by the presence of Shapiro steps.

The last two systems are occasions to frame the Josephson effect in other contexts of solid state physics: on one hand in granular superconducting systems, the interplay between Josephson, Anderson, Kondo and Mott physics, on the other nanoclustering and Josephson coupling as a key for very high temperature superconductivity.

\section{About My Story with the Josephson Effect}

I do not have any direct personal memories to recall in my contribution, since I never met Prof. Brian Josephson, but I am aware that my scientific life has been better and happier with the Josephson effect. My story started in the very late 1980s when I had to choose the topic for my master thesis and I was extremely lucky to meet Antonio Barone and to work with him for many years. Antonio used to transfer to all his students in Napoli some kind of ethos on the Josephson effect that still survives. He made Napoli a special place where to carry out research on superconductivity, and especially on the Josephson effect.

Antonio Barone could have told the story of how the Josephson effect has also spread here in Napoli and solidly established itself since the very beginning of the 1970s. I am too inadequate for that and I prefer to pick a topic that has characterized research efforts on the Josephson effect here in Napoli, and more specifically I will tell my part of the story.

I start from these two sentences from the review paper on Superconducting phase qubits of John Martinis [9] "Superconducting qubit research began in the 1980s motivated by the question, posed by Anthony Leggett, whether macroscopic variables would behave in a quantum mechanical fashion. Initial experiments verified quantum behavior via the phenomenon of tunneling out of the zero-voltage state of a current-biased Josephson junction. At UC Berkeley, quantum mechanical behavior was also demonstrated by the existence of quantized energy levels. This observation provided stronger proof of quantum behavior, and established at an early stage (before the ideas of qubits were even widely established) that superconducting circuits could be used as general quantum systems."

Macroscopic Quantum Tunneling (MQT) [10, 11] has thus been fundamental and is of great actuality also for superconducting qubits. This is the ultimate experiment one wants to do on a junction to fully understand its physics. As a tribute to Prof. Brian Josephson's for his 80s on my side I will sketch some ideas on the theme of MQT in unconventional systems. MQT is the first manifestation of secondary quantum effects in Josephson junctions. For unconventional systems the observation of MQT says that despite the unknown nature of the junction or its imperfections which transform the unconventional junction into some kind of "black box," the junction keeps a robust internal quantum coherence. This is also the first feeling one gets on real dissipation, once the junction is in a circuit and is exposed to environmental noise. This work builds on a series of experiments on well-known conventional JJs culminated in the milestone work in the field realized at UC Berkeley $[12,13]$. 
I will mostly confine my story on experiments on high critical temperature superconductor (HTS) JJs. HTS oxides enlarge the occurrence of superconductivity and of the Josephson effect to unexpected energy and length scales. A supercurrent between two phase coherent electrodes up to about $100 \mathrm{~K}$ is the first remarkable example. Dimensionality, d-wave order parameter (OP) symmetry, strong correlations,... contribute to set the phenomenology observed in HTS JJs, which still embody the example of what one expects from unconventional junctions. The concepts and know-how matured in handling HTS JJs have been fundamental for the design of junctions based on most of the unconventional superconductors discovered after HTS.

Grain boundaries (GBs) have offered a versatile tool to have access to the Josephson effect in HTS, taking advantage of a significant reduction of the critical current between two grains with different orientations, which generates weak coupling and Josephson-like behavior between the two electrodes in some limits [14-16]. The biepitaxial (BP) GB technique uses changes of the orientation of HTS films induced by epitaxial growth on structured template layers. In the configuration developed in Napoli $\mathrm{YBa}_{2} \mathrm{Cu}_{3} \mathrm{O}_{7-x}$ grows along the [001] direction on the $\mathrm{MgO}$ and on the $\mathrm{CeO}_{2}$ seed layers, while it grows along the [103]/[013] direction on $\mathrm{SrTiO}_{3}$ substrates [17]. The presence of the $\mathrm{CeO}_{2}$ produces an additional $45^{\circ}$ in-plane rotation of the $Y B C O$ axes with respect to the in-plane directions of the substrate [18]. The out-of-plane tilt was the main ingredient for the success of this type of junction providing a more selective and less transmissive transport regime. This was the key for the realization of a couple of important experiments in the field $[16,19]$.

In the former the dependence of the Josephson effect on the relative orientation of the junction and the crystal axes of the superconducting electrodes, of particular relevance for systems with an anisotropic pair wave function, was exploited. Evidence of an oscillatory dependence of the critical current $\mathrm{I}_{C}$ as a function of the angle between the GB line and the (001) in-plane direction of the substrate was found in remarkable agreement with theoretical predictions based on $\mathrm{d}_{x^{2}-y^{2}}$-wave pairing symmetry. Even if $\mathrm{d}_{x^{2}-y^{2}}$ wave order parameter (OP) was already solidly established thanks to the two corner-stone experiments by the Urbana and IBM groups (see, for instance, the reviews with all appropriate references $[20,21])$, these measurements demonstrated for the first time that "intrinsic" d-wave effects are dominant in the phenomenology of all-HTS Josephson junctions themselves (not inserted in any loop) independent of the interface details.

In the latter experiment realized within a collaboration between Chalmers University and the University of Napoli, MQT was for the first time observed in an unconventional JJ $[22,23]$, other than those based on classical superconductors and layouts. MQT was revealed in the most unfavorable configuration, for a BP junction with a lobe of the OP facing a node, thus in presence of low energy quasi-particles. The escape rate of the superconducting phase $\varphi$ from a local minimum in the washboard potential into the running state as a function of temperature was studied using the same techniques as MQT experiments on low- $T_{C}$ junctions $[12,13,24]$. The switching current probability distributions as a function of temperature substantially follows what commonly measured on LTS JJs, with a saturation of the measured standard deviation $\sigma$ below $50 \mathrm{mK}$ [22], which corresponds to the crossover temperature $T_{c r}$ from the thermal to the MQT regime. Macroscopic energy quantization in the presence of radio-frequency radiation was also later demonstrated [23].

In later studies junction parameters were finely tuned to explore phase dynamics in the moderately damped regime, commonly reported as the phase diffusion (PD) regime [2529]. $\left(\mathrm{La}_{0.3} \mathrm{Sr}_{0.7}\right)\left(\mathrm{Al}_{0.65} \mathrm{Ta}_{0.35}\right) \mathrm{O}_{3}$ (LSAT) substrates tend to reduce the stray capacitance and change the overall capacitance of the junction. The fall off of $\sigma$ in the PD regime provides a tool for an accurate estimation of the dissipation in the moderately damped regime through a fitting procedure based on Monte Carlo simulations [28-31]. These experiments suggest that also for HTS JJs phase dynamics can be finely tuned in a wide range of junction parameters. We have been always fascinated by the idea that these findings are robust with respect to faceting and to the presence of significant impurities and disorder along the barriers and in the electrodes, confirming a protected global coherence independent of the poor barrier quality.

HTS GB JJs can also offer junctions with relatively higher critical current density $\mathrm{J}_{c}[15,16]$. For these junctions standard phase dynamics collapses and cannot be sustained above some threshold of $\mathrm{J}_{c}$ [32]. Here non-equilibrium dynamics play a major role and can be partly codified in the local heating process. This is of great relevance for all the experiments using nanowires or, more generally, low-dimensional barriers, which should be concerned about possible heating effects. These methods have been applied to other unconventional Josephson systems, as those with ferromagnetic barriers [33].

The Josephson effect has accompanied most of my scientific life from Napoli to Berkeley and Stony Brook, from Yorktown back to Napoli. As a student, as a post-doc, as a researcher, as a professor and advisor of several students, I have lived so many moments of "joy of knowledge" that are connected to the Josephson effect. The Josephson effect has also been the direct or indirect opportunity to meet many great colleagues all over the world. It would be impossible to acknowledge all of them. My deep gratitude goes to those I have worked with day by day in these years: from the very beginning till a few years ago Antonio 
Barone; John Kirtley, Floriana Lombardi, Giampiero Pepe, Arturo Tagliacozzo and the new generation Davide Massarotti, Domenico Montemurro, Procolo Lucignano, Daniela Stornaiuolo, Luigi Longobardi.

If I look at the future, apart from the ultimate dreams of understanding superconductivity in HTS and programming a superconducting quantum computer, my secret scientific dream is the perfect Josephson junction, possibly operating at high temperatures. A perfect junction would be also a key for improving qubit performances [34-37]. Much remains to be done and this is fun! Happy Birthday Professor Josephson!

\section{References}

1. Josephson, B.D.: Possible new effects in superconductive tunnelling. Phys. Lett. 7, 251-253 (1962)

2. Josephson, B.D.: Supercurrents through barriers. Adv. Phys. 14, 419-451 (1965)

3. Barone, A.: Paternó Physics and Applications of the Josephson Effect. Wiley, New York (1982)

4. Likharev, K.K.: Dynamics of Josephson Junctions and Circuits. Gordon and Breach, New York (1986)

5. Kulik, I.O., Yanson, I.K.: Josephson Effect in Superconducting Tunnel Structures(1970) (Russian Nauka, Moscow, 1970) (1972). English Keter Press, Jerusalem (1972)

6. Van Duzer, T., Turner, C.W.: Principles of Superconductive Devices and Circuits. Elsevier, New York (1981)

7. Wolf, E., Arnold, G., Gurvitch, M., Zasadzinski, J. (eds.): Josephson Junctions History, Devices, and Applications. Pan Stanford Publishing, Singapore (2017)

8. Tafuri, F. (ed.): Fundamentals and Frontiers of the Josephson Effect. Springer Nature Swizterland, Cham (2019)

9. Martinis, J.M.: Superconducting phase qubits. Quantum Inf. Process 8, 81-103 (2009)

10. Caldeira, A.O., Leggett, A.J.: Quantum tunnelling in a dissipative system. Annals of Physics 149, 374-456 (1993)

11. Caldeira, A.O., Leggett, A.J.: Influence of dissipation on quantum tunneling in macroscopic systems. Phys. Rev. Lett. 46, 211 (1981)

12. Devoret, M.H., Martinis, J.M., Clarke, J.: Measurements of macroscopic quantum tunneling out of the zero-voltage state of a currentbiased Josephson junction. Phys. Rev. Lett. 55, 1908 (1985)

13. Martinis, J.M., Devoret, M.H., Clarke, J.: Experimental tests for the quantum behavior of a macroscopic degree of freedom: The phase difference across a Josephson junction. Phys. Rev. B 35, 4682 (1987)

14. Chaudari, P., Mannhart, J., Dimos, D., Tsuei, C.C., Chi, C.C., Oprysko, M.M., Scheuermann, M.: Direct measurement of the superconducting properties of single grain boundaries in $Y_{1} \mathrm{Ba}_{2} \mathrm{Cu}_{3} \mathrm{O}_{7-\delta}$. Phys. Rev. Lett. 60, 1653 (1988)

15. Hilgenkamp, H., Mannhart, J.: Grain boundaries in high- $T_{c}$ superconductors. Rev. Mod. Phys. 74, 485 (2002)

16. Tafuri, F., Kirtley, J.R.: Weak links in high critical temperature superconductors. Rep. Prog. Phys. 68, 2573 (2005)

17. Tafuri, F., Miletto Granozio, F., Carillo, F., Di Chiara, A., Verbist, K., Van Tendeloo, G.: Microstructure and Josephson phenomenology in $45^{\circ}$ tilt and twist $\mathrm{YB}_{2} \mathrm{Cu}_{3} \mathrm{O}_{7-\delta}$ artificial grain boundaries. Phys. Rev. B 59, 11523-11531 (1999)
18. Lombardi, F., Tafuri, F., Ricci, F., Miletto Granozio, F., Barone, A., Testa, G., Sarnelli, E., Kirtley, J.R., Tsuei, C.C.: Intrinsic $d$-wave effects in $\mathrm{YBa}_{2} \mathrm{Cu}_{3} \mathrm{O}_{7-x}$ grain boundary Josephson junctions. Phys. Rev. Lett. 89, 207001-207004 (2002)

19. Tafuri, F., Massarotti, D., Galletti, L., Stornaiuolo, D., Montemurro, D., Longobardi, L., Lucignano, P., Rotoli, G., Pepe, G.P., Tagliacozzo, A., Lombardi, F.: Recent achievements on the physics of High-Tc superconductor Josephson junctions: background, perspectives and inspiration. J. Supercond. Novel Magn. 26, 21-41 (2013)

20. Van Harlingen, D.J.: Phase-sensitive tests of the symmetry of the pairing state in the high-temperature superconductors ${ }^{-}$Evidence for $d_{x^{2}-y^{2}}$ symmetry. Rev. Mod. Phys. 67, 515 (1995)

21. Tsuei, C.C., Kirtley, J.R.: Pairing symmetry in cuprate superconductors. Rev. Mod. Phys. 72, 969 (2000)

22. Bauch, T., Lombardi, F., Tafuri, F., Barone, A., Rotoli, G., Delsing, P., Claeson, T.: Macroscopic quantum tunneling in $d$ Wave $\mathrm{YBa}_{2} \mathrm{Cu}_{3} \mathrm{O}_{7-x}$ Josephson junctions. Phys. Rev. Lett. 94, 087003 (2005)

23. Bauch, T., Bauch, T., Lindström, T., Tafuri, F., Rotoli, G., Delsing, P., Claeson, T., Lombardi, F.: Quantum dynamics of a d-wave Josephson junction. Science 311, 57-60 (2006)

24. Massarotti, D., Tafuri, F.: Phase dynamics and macroscopic quantum tunneling. In: Tafuri, F. (ed.) Fundamentals and Frontiers of the Josephson Effect, pp. 455-512. Springer Nature Swizterland (2019). Chapter 11

25. Kautz, R.L., Martinis, J.M.: Noise-affected I - V curves in small hysteretic Josephson junction. Phys. Rev. B 42, 9903 (1990)

26. Kivioja, J.M., Nieminen, T.E., Claudon, J., Buisson, O., Hekking, F.W.J., Pekola, J.P.: Observation of transition from escape dynamics to underdamped phase diffusion in a Josephson junction. Phys. Rev. Lett. 94, 247002 (2005)

27. Männik, J., Li, S., Qiu, W., Chen, W., Patel, V., Han, S., Lukens, J.E.: Crossover from Kramers to phase-diffusion switching in moderately damped Josephson junctions. Phys. Rev. B 71, 220509 (2005)

28. Longobardi, L., Massarotti, D., Rotoli, G., Stornaiuolo, D., Papari, G., Kawakami, A., Pepe, G.P., Barone, A., Tafuri, F.: Thermal hopping and retrapping of a Brownian particle in the tilted periodic potential of a NbN/MgO/NbN Josephson junction. Phys. Rev. B 84, 184504 (2011)

29. Massarotti, D., Longobardi, L., Galletti, L., Stornaiuolo, D., Montemurro, D., Pepe, G.P., Rotoli, G., Barone, A., Tafuri, F.: Escape dynamics in moderately damped Josephson junctions, Low Temp. Phys. 38, 263-272 (2012)

30. Longobardi, L., Massarotti, D., Stornaiuolo, D., Galletti, L., Rotoli, G., Lombardi, F., Tafuri, F.: Direct transition from quantum escape to a phase diffusion regime in $\mathrm{YBaCuO}$ Biepitaxial Josephson junctions. Phys. Rev. Lett. 109, 050601 (2012)

31. Longobardi, L., Massarotti, D., Rotoli, G., Stornaiuolo, D., Papari, G., Kawakami, A., Pepe, G.P., Barone, A., Tafuri, F.: Quantum crossover in moderately damped epitaxial $\mathrm{NbN} / \mathrm{MgO} / \mathrm{NbN}$ junctions with low critical current density. Appl. Phys. Lett. 99, 062510-062512 (2011)

32. Massarotti, D., Stornaiuolo, D., Lucignano, P., Galletti, L., Born, D., Rotoli, G., Lombardi, F., Longobardi, L., Tagliacozzo, A., Tafuri, F.: Breakdown of the escape dynamics in Josephson junctions. Phys. Rev. B 92, 054501 (2015)

33. Massarotti, D., Pal, A., Rotoli, G., Longobardi, L., Blamire, M.G., Tafuri, F.: Macroscopic quantum tunneling in spin filter ferromagnetic Josephson junctions. Nat. Commun. 6, 7376 (2015)

34. Clarke, J., Wilhelm, F.K.: Superconducting quantum bits. Nature 453, 1031-1042 (2008) 
35. Devoret, M.H., Martinis, J.M.: Superconducting qubits. In: Esteve, D., Raimond, J.M., Dalibard, J. (eds.) Quantum entanglement and information processing. volume 79 of Les Houches Summer School Session, pp. 443-485 (2004). Les Houches Session 79th on Quantum Entanglement and Information Processing; K. Osbourne and J. M. Martinis, "Superconducting qubits and the physics of Josephson junctions", ibidem
36. McDermott, R.: Materials origins of decoherence in superconducting qubits. IEEE Trans. Appl. Cond. 19, 2-13 (2009)

37. Devoret, M.H., Schoelkopf, R.J.: Superconducting circuits for quantum information: an outlook. Science 339, 1169-1174 (2013)

Publisher's Note Springer Nature remains neutral with regard to jurisdictional claims in published maps and institutional affiliations. 